DOI: https://doi.org/10.14311/TPFM.2022.014

\title{
ASSESSMENT OF VARIOUS CONVERGING INLET NOZZLES FOR WIND TUNNEL USING CFD
}

\author{
P. Kosiak, T. Radnic, M. Mamula, J. Hála, D. Šimurda, M. Luxa \\ Department of Fluid Dynamics, Institute of Thermomechanics of the Czech Academy of \\ Sciences, Dolejškova 1402/5, 18200 Prague, Czech Republic
}

\begin{abstract}
A new calibration tunnel intended for pressure probe calibration is being designed at the Aerodynamic Laboratory of the Institute of Thermomechanics of the Czech Academy of Sciences (IT CAS). A critical part of the design is the converging nozzle since it can substantially affect the resulting flow field in the test section. Three types of nozzles were chosen for the CFD investigation: two-sine, Vitoshinski, and Vagt. For each type of nozzle, two configurations were considered: long $(645 \mathrm{~mm})$ and short $(353 \mathrm{~mm})$. Flow of the viscous, compressible fluid through all the variants was simulated using Ansys CFX commercial software. Results proved the Vagt nozzle to have the most uniform velocity profile and zero curvature at its end making it the most suitable for the intended use.
\end{abstract}

Keywords: CFD, ANSYS CFX, converging nozzle, wind tunnel design

\section{Introduction}

For aerodynamic measurements using pressure probes a reliable probe calibration is required. To obtain such calibrations at the Aerodynamic Laboratory of the IT CAS, it was decided to build a new calibration tunnel designated specifically for this purpose. The aim of the presented investigation is to find the optimal configuration of the converging inlet nozzle section, which represents a substantial part of the whole calibration wind tunnel. Throughout this investigation, three different types of nozzles were considered and evaluated: the Vitoshinski nozzle [1], two-sine nozzle [2] and Vagt nozzle [3]. Each type was investigated in two configurations varying in length: short $(353 \mathrm{~mm})$ and long $(645 \mathrm{~mm})$. All nozzles were evaluated for the same inlet diameter of $300 \mathrm{~mm}$ and outlet diameter of $150 \mathrm{~mm}$.

Ansys CFX commercial software utilizing the finite volume method for the discretization of the governing Navier-Stokes equations and RANS approach for turbulence modelling was utilized for the simulation of the flow through all six variants of nozzles.

\section{Geometry of nozzles}

Meridional shape of each nozzle's meridional section is defined by a different function.

Equation (1) defines meridional shape of the Vitoshinski nozzle:

$$
r_{(x)}=\frac{r_{2}}{\sqrt{1-\left[1-\left(\frac{r_{2}}{r_{1}}\right)^{2}\right] \frac{\left(1-\frac{x^{2}}{L^{2}}\right)^{2}}{\left(1+\frac{x^{2}}{3 L^{2}}\right)^{3}}}}
$$

Equation (2) defines meridional shape of the Vagt nozzle:

$$
r_{(x)}=\frac{x^{3}}{L^{3}}\left(2-\frac{x^{3}}{L^{3}}\right)\left(r_{1}-r_{2}\right)+r_{2},
$$

In equations (1) and (2), $r_{1}$ and $r_{2}$ are inlet and outlet radii, respectively, $r_{(x)}$ is the arbitrary radius of the nozzle, $L$ is the length of the nozzle and $x$ is the distance between inlet cross-section and cross-section with radius $r$. 
For analytical description, meridional shape function of the two-sine nozzle is divided into 2 parts. The first part is described by equations (3) and (4):

$$
\begin{aligned}
& x=q \cdot \cos (\beta)+\tan ^{2}(\beta) \frac{L}{3 \pi} \sin \left[q \frac{3 \cdot \pi}{L} \cos (\beta)\right], \\
& r=\frac{d_{1}}{2}-q \cdot \sin (\beta)+\tan (\beta) \frac{L}{3 \pi} \sin \left(q \frac{3 \pi}{L} \cos (\beta)\right),
\end{aligned}
$$

where $q$ is in the range $[0 ; L /(3 \cdot \cos (\beta))]$.

The second part is described by equations (5) and (6):

$$
\begin{gathered}
x=q \cdot \cos (\beta)+\tan ^{2}(\beta) \frac{2 L}{3 \pi} \sin \left[\frac{3 \pi}{2 L} \cos (\beta)\left(q+\frac{L}{3 \cdot \cos (\beta)}\right)\right], \\
r_{(q)}=\frac{d_{1}}{2}-q \cdot \sin (\beta)+\tan (\beta) \frac{2 L}{3 \pi} \sin \left[\frac{3 \pi}{2 l} \cos (\beta)\left(q+\frac{L}{3 \cdot \cos (\beta)}\right)\right],
\end{gathered}
$$

where $q$ is in the range $[L /(3 \cos (\beta)) ; L / \cos (\beta)]$. L is the length of the nozzle, and it is for this case defined by equation (7):

$$
L=\frac{d_{1}-d_{2}}{2 \tan (\beta)}
$$

where $\beta$ is the angle between the line intersecting the beginning and ending points of the meridional curve and the axis of the nozzle. The angle $\beta$ should be lower than $23^{\circ} . d_{1}$ and $d_{2}$ are inlet and outlet diameters, respectively.

Each nozzle was investigated in two configurations: short $(353 \mathrm{~mm})$ and long $(645 \mathrm{~mm})$. The inlet radius for all six nozzles was $300 \mathrm{~mm}$ and the outlet radius was $150 \mathrm{~mm}$. Meridional shapes of these nozzles in meridional section are shown in Figure 1 . The angle $\alpha$, inverse tangent of the curve slope is shown in Figure 2. Figure 3 shows the derivative of the angle $\alpha$, which is related to curvature. The axial coordinate is represented by the dimensionless value $x / L$, therefore both short and long nozzle share the same coordinates. In Figure 1 it is clearly visible that within the interval $x / L \in[0 ; 0.1]$ the two-sine nozzle converges at much slower rate than the other two nozzles. Figure 3 also shows that the two-sine nozzle has much higher curvature than Vitoshinski and Vagt nozzle at axial position at approximately $x / L=0.2$. At the same time, there is a discontinuity in curvature at around $x / L=0.34$ in the case of the two-sine nozzle. The curvature of the Vitoshinski and Vagt nozzles is lower than two-sine nozzle at axial position within the interval $x / L \in[0.5 ; 0.9]$. Summarization can be made that the two-sine nozzle has slower onset contraction, then it has very high curvature and discontinuous curvature change in the inflection point. At the same time, it has higher curvature in the region of decreasing convergence. It is also worth noting, that the Vitoshinski nozzle has non-zero curvature at its end while other two nozzles have a smooth transition to the straight part downstream the contraction.

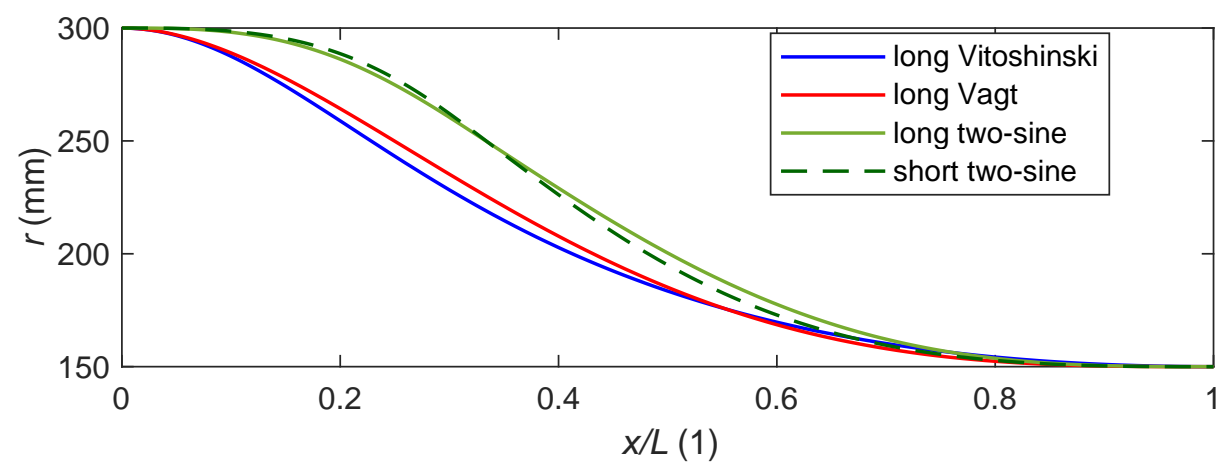

Figure 1: Meridional shape function for each investigated nozzle. 


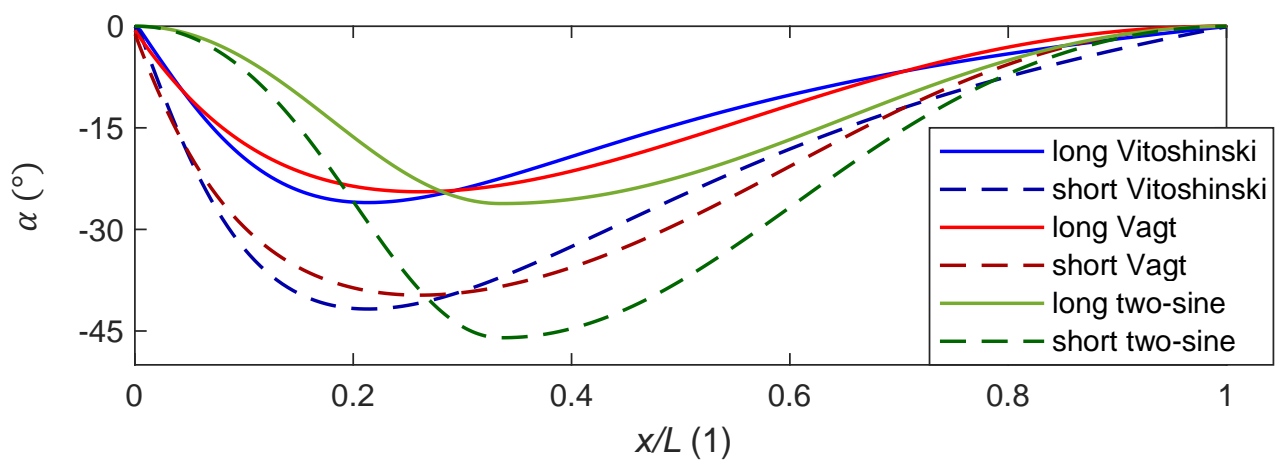

Figure 2: Distribution of the angle $\alpha$ of the meridional shape function.

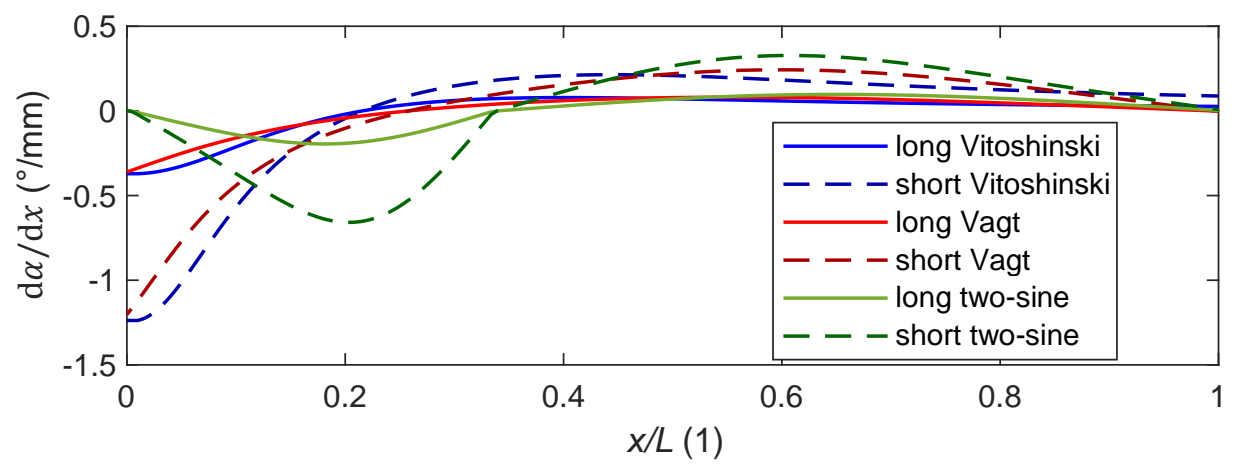

Figure 3: Distribution of the first derivative of the angle $\alpha$ of the meridional shape function.

\section{Numerical simulation}

The computational domain is axisymmetric quarter cut of a converging nozzle and $1 \mathrm{~m}$ long straight part downstream the contraction. The simulation was initially performed with the large inlet area followed by rounded transition to the actual nozzle. However, the radial velocity induced in the flow in the inlet part caused flow separation in shorter configuration of nozzles. Moreover, the nozzle is intended to be mounted on the straight part, so such simulation would not model the real use case of the nozzle. Thus, the inlet part of the computational domain was left as plane of the nozzle origin.

In total, six variants of nozzles were modelled; the $353 \mathrm{~mm}$ variant named 'short', and $653 \mathrm{~mm}$ variant named 'long'. The computational meshes were created in ICEM CFD commercial software. By adding the straight part downstream of the nozzle, it enabled us to avoid the possible non-physical influence of the outlet boundary conditions on the flow field inside the nozzle. The quarter cut of the computational domain required symmetry boundary condition to be used.

Numerical simulation was conducted in Ansys CFX commercial software The flow was modelled as a flow of viscous compressible fluid [4] and the flow medium was set to be air with ideal gas properties.

For modelling of turbulent viscosity, the Shear Stress Transport (SST) fully turbulent model was chosen. This model of turbulence is combination of two turbulent models: $k-\varepsilon$ in the free stream and $k-\omega$ close to the walls [5].

The solver boundary conditions were set as follows. The inlet total pressure $p_{t o t}=101325 \mathrm{~Pa}$ was set constant at the inlet. Inlet total temperature was set as $T_{\text {tot }}=288 \mathrm{~K}$, and the turbulence intensity $T u_{1}=$ $1 \%$. The outlet was set as a subsonic pressure outlet with static pressure $p_{2}=94303 \mathrm{~Pa}$. The wall of the nozzle and straight part downstream was modelled as no-slip adiabatic smooth wall. The quarter cut sides of the domain had the symmetry boundary condition set, as the rotational periodicity conditions did introduce non-physical phenomena in the flow. Thus, governing equations were solved in Cartesian coordinate system. Investigation of origin of said phenomena is outside the scope of this project. Meridional cut view of the mesh is shown in Figure 4. The mesh for short variant of nozzles contained 349448 hexahedral cells and mesh for long nozzles contained 491986 hexahedral cells. The mesh was generated using the blocking $\mathrm{O}$-grid technique. 


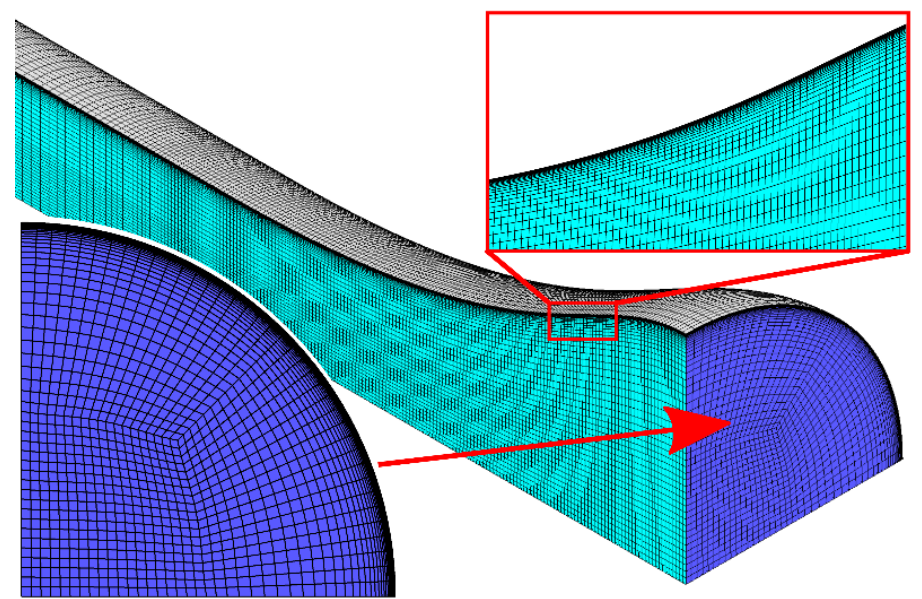

Figure 4: Computational domain with mesh on boundaries and detail of near wall refinement.

For the more accurate simulation of the boundary layer, the mesh in the vicinity of the wall was refined so as the non-dimensional wall distance of the first cell $y+$ was lower than 1 . The refinement is shown in upper left corner of Figure 4. The criterion of the convergence for the simulation was maximum values of residuals of the momentum and turbulent kinetic energy. Maximum values of residuals were considered, and simulation was deemed converged when the maximal residual dropped below $\operatorname{Res}_{M}<10^{-7}$.

\section{Results}

The evaluation of individual nozzle qualities started with the evaluation of velocity profile at the exit plane of the nozzles. The velocity profiles and their development are shown in Figure 5 for short configurations of nozzles and in Figure 6 for long configurations. The short configurations have higher curvature in order to retain the contraction factor of 2 (ratio of the inlet to outlet diameter $d_{1} / d_{2}$ ). As a result, the flow near the wall of short configurations has more rapid acceleration than the flow near the wall of long nozzles. This means that the velocity profiles of the long configurations are more homogenous compared to the profiles of short configurations. The criterion of evaluation of the quality of the nozzle is the velocity profile at the outlet. It is apparent that the outlet velocity profile of Vagt nozzle in the long configuration has the most uniform shape, hence the least variation of the velocity of the free stream at the $x / L=1$.

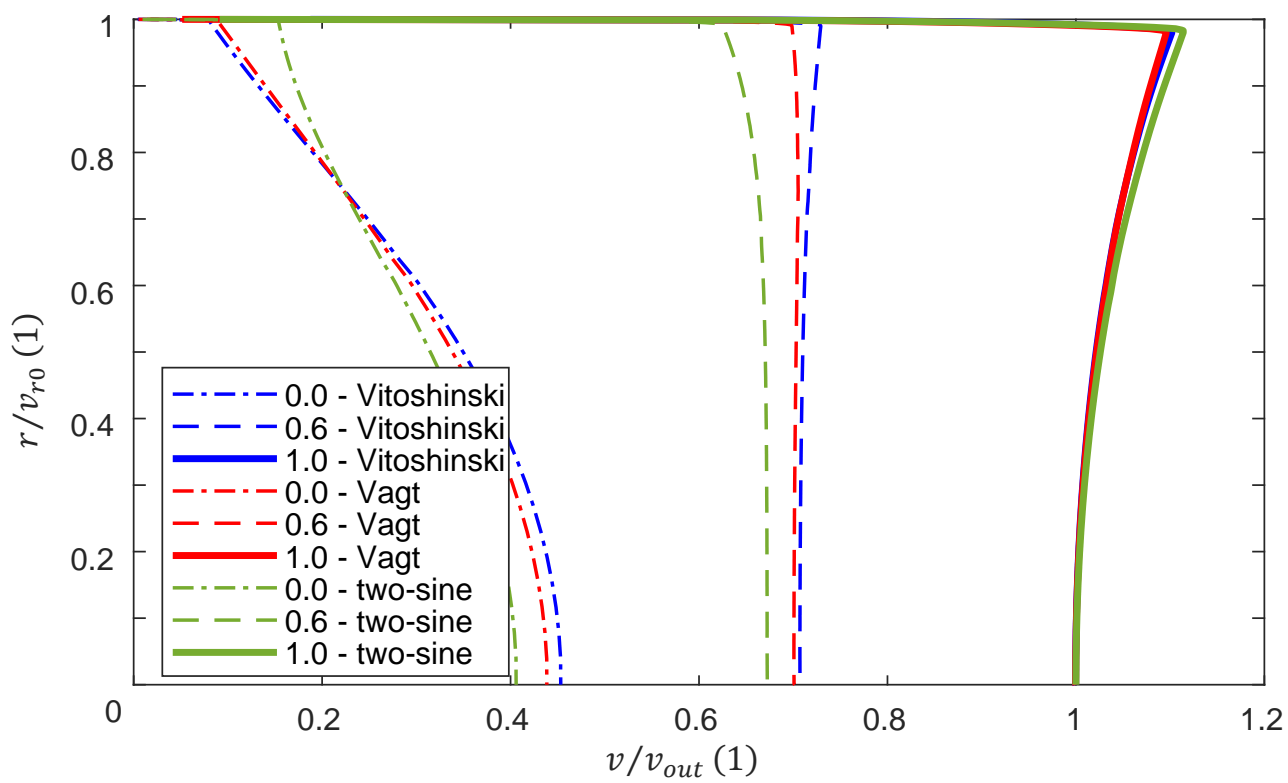

Figure 5: Velocity profiles at locations $x / L=[0 ; 0.6 ; 1.0]$ for the short configurations of the nozzles 


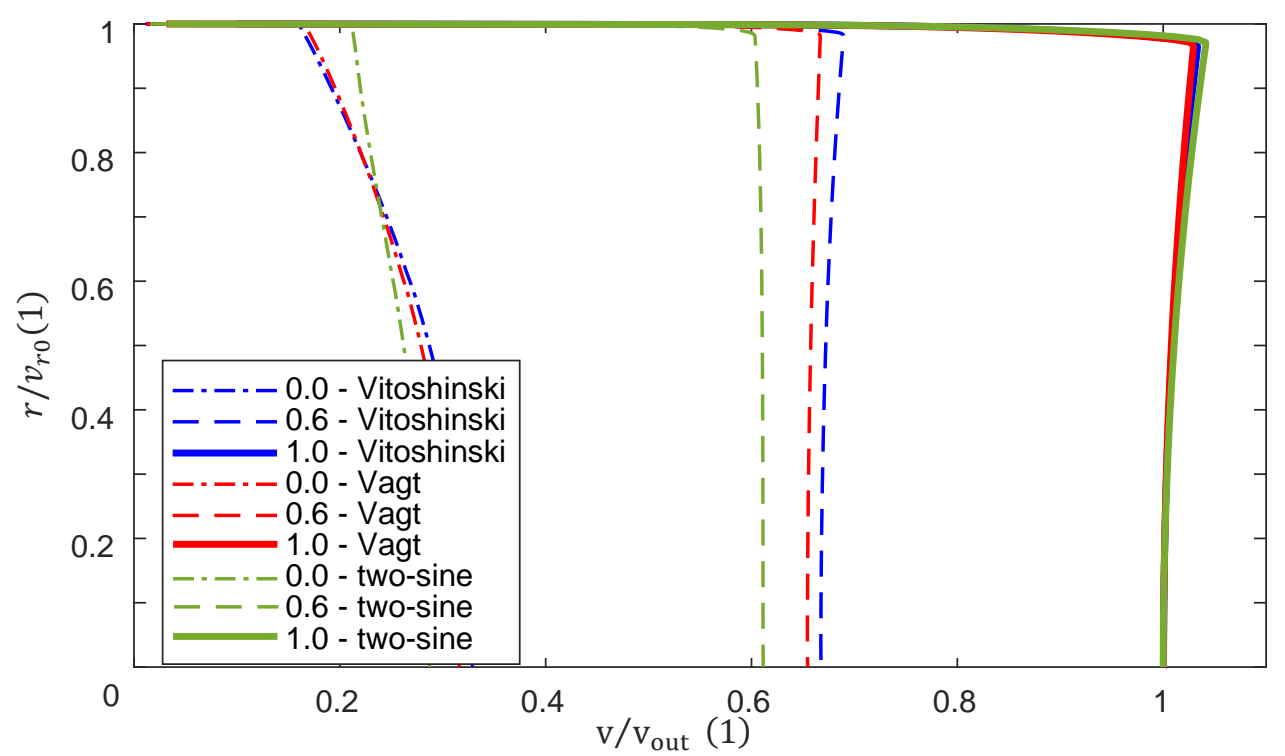

Figure 6: Velocity profiles at locations $x / L=[0 ; 0.6 ; 1.0]$ for the long configurations of the nozzles

The distribution of skin friction $c_{f}$ along the wall is shown in Figure 7. The skin friction coefficient is calculated as a function of the wall shear stress obtained directly from the numerical solver and the velocity of the flow as seen in equation (8).

$$
c_{f}=\frac{\tau}{\frac{1}{2} \rho v^{2}}
$$

This distribution shows that the values of $c_{f}$ for all variants of nozzles extremely decrease close to the location $x / L=0.1$. The reason of this behaviour is the considerable curvature of the nozzle meridional shape. The biggest decrease of $c_{f}$ is characteristic of short nozzles which have a larger curvature to provide contraction value of 2 on a shorter distance. The values $c_{f}$ which are close to 0 mean that a separation of flow could have occurred or that there is significant risk of flow separation during operation in certain flow regimes. Long configurations of the nozzles naturally do not suffer from the separation risk because of lower curvature and more favourable pressure gradients. Then growth of the $c_{f}$ for all nozzles is observed in the interval between $x / L=0.1$ and $x / L=0.7$. The reason for this growth is the increase of the velocity near the wall of the nozzle, which is the result of the curvature and decrease of the cross-section of the nozzle. The higher values of $c_{f}$ are characteristic for the short configuration of the two-sine nozzle, short configuration of the Vagt nozzle and long configuration of the two-sine nozzle. The lower values of $c_{f}$ are characteristic for both configurations of the Vitoshinski nozzle and for long configuration of the Vagt nozzle. Increase of the $c_{f}$ corresponds to the increase of the velocity because the shear stress is dependent on the velocity. As a result, long Vitoshinski and Vagt nozzles have the best shear stress characteristics compared to the other investigated nozzles. 


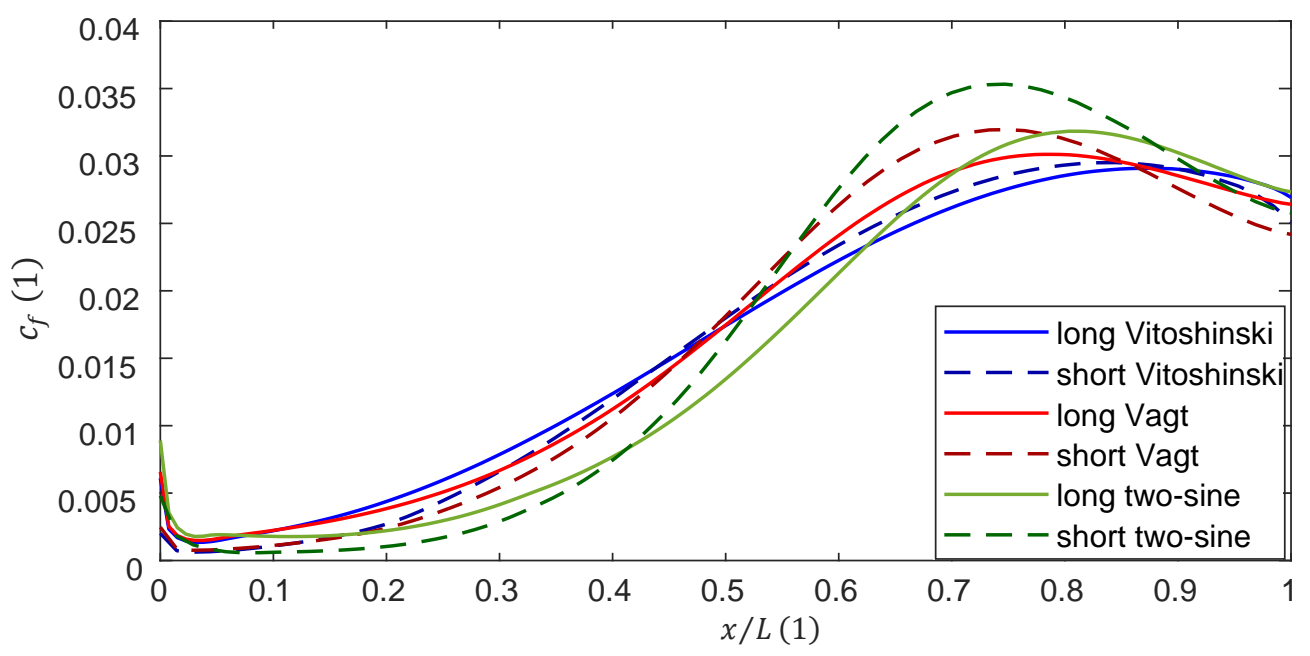

Figure 7: Distribution of the skin friction coefficient along the wall of the nozzle.

The analysis of Figure 8 shows that the long and short variants of two-sine nozzle have abnormally high displacement thickness of the boundary layer at axial position around $x / L=0.18$. The reason of this increase is the low curvature and acceleration in the interval $0<x / L<0.18$. Both short and long configurations of the two-sine nozzles exhibit decrease of the displacement thickness in the interval $0.18<x / L<0.5$, which is the result of more rapid acceleration of the flow caused by a sharp decrease of the cross-section surface. In the region of $x / L>0.5$, the cross section surface area decreases less rapidly and as a result the acceleration of the flow slows down, which in turn causes the increase of the boundary layer displacement thickness. The short configurations of the two-sine nozzle have more rapidly decreasing cross-section surface area near the outlet compared with the long configuration of the same nozzle. This causes the higher displacement thickness at the outlet of the longer configuration of the two-sine nozzle compared to the short one.

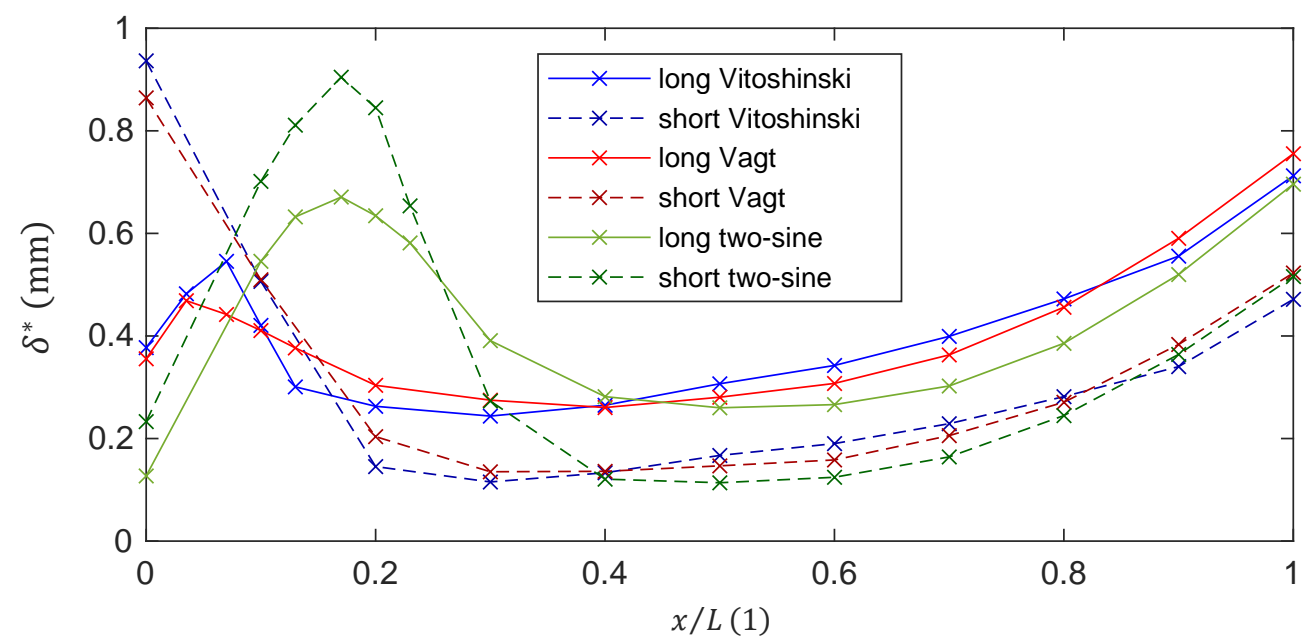

Figure 8: Distribution of the boundary layer displacement thickness

The behaviour of other types of nozzles is more similar one to another. Vitoshinski and Vagt nozzles show decrease of the displacement thickness in the region $0<x / L<0.3$ and $0<x / L<0.4$ respectively. This also corresponds to the increase of the displacement thickness in the region $0.3<x / L<1$ and $0.4<x / L<1$. The reason for the decreasing displacement thickness of the boundary layer is the high acceleration of the flow. Increase of the displacement thickness can be attributed to the lower acceleration of the flow. The higher displacement thickness of the boundary layer at the outlet of the long configurations 
of the nozzles compared with the displacement thickness for the short configurations is the result of a greater length and hence longer distance on which the boundary layer grows.

The loss of the kinetic energy inside the nozzle is represented by the loss coefficient defined by equation (9), see [6].

$$
\zeta=1-\frac{T_{t o t}-T_{2}}{T_{t o t}-T_{2 i s}}
$$

where $T_{\text {tot }}$ is the total temperature, $T_{2}$ is static temperature at the outlet and $T_{2 i s}$ is isentropic static temperature at the outlet.

The values of the loss coefficient, which are shown in Table 1, show that the longer configurations seem to have higher values of the loss coefficient. The explanation of the higher values of the loss coefficient might be in larger area of the nozzle wall at which losses originate due to the no-slip boundary condition. The short configuration of the two-sine nozzle has the lowest value of loss coefficient and the long Vagt has the highest values of the loss coefficient. It is also worth noting that the loss coefficient is very low for all the evaluated nozzles.

Table 1 - list of the Loss coefficient values for different nozzles

\begin{tabular}{|c|c|}
\hline & Loss Coefficient $\zeta(\%)$ \\
\hline short two-sine nozzle & 0.809517 \\
\hline long two-sine nozzle & 1.139374 \\
\hline short Vagt & 0.829324 \\
\hline long Vagt & 1.256685 \\
\hline short Vitoshinski & 0.810439 \\
\hline long Vitoshinski & 1.210781 \\
\hline
\end{tabular}

\section{Discussion}

The quality of the flow through the three nozzles, each in two length configurations, was evaluated. The conclusions are drawn with respect to the intended use of the nozzle, which is the converging part of the calibration wind tunnel. During the calibration of any probe, the most important parameter is the axial velocity of the flow at the outlet and any significant non-homogenous flow phenomena. It is also worth mentioning that the nozzles will be operated at different regimes than just the investigated one. More thorough investigation is, however, out of scope of this paper.

From the defined point of view, the potential of flow separation is very undesirable. The distribution of skin friction in Figure 7 shows that short configuration of the two-sine nozzle has very low skin friction coefficient $c_{f}$ in the area up to $x / L<0.2$. This means that there is a very low wall shear, and that the flow separation might occur in different flow regime. The graph in Figure 1 shows, that only Vitoshinski nozzle has non-zero curvature at the outlet of the nozzle. This could in certain regimes cause unfavourable pressure gradients in the boundary layer.

Regarding the flow field velocity profile at the outlet of the nozzle, the short configurations of the nozzles exhibit quite non-uniform velocity profiles at their ends. The difference shown in Figure 5 means that flow closer to the wall is much faster than at the axis of the nozzle. The longer configurations, on the contrary, show very uniform velocity profiles. In any case, the uniform velocity profile is favourable for the calibration test section downstream of the nozzle.

The shorter configurations proved to be more efficient and having thinner boundary layer. The graph in Figure 8 shows that the shorter configurations do not form boundary layers as thick as longer configurations. This is to be expected due to the length of the nozzle and the boundary layer having more time to thicken as well as less rapid acceleration of the flow which would decrease the boundary layer thickness.

The most promising candidate for the inlet nozzle of the new calibration wind tunnel seems to be the long configuration of the Vagt nozzle. It has zero curvature at the end, more consistent flow acceleration along the nozzle and does not show risk of forming of flow separations.

However, different use cases would deem different nozzles as most favourable. Should efficiency have been the most concerning parameter and outlet flow field would not be of significance for the application, probably the short configuration of the Vitoshinski nozzle would be the best candidate. 


\section{Conclusions}

The evaluation of the flow field quality in different three types of converging nozzles was performed using Ansys CFX commercial software with fully turbulent SST turbulence model. Each of the three geometries were investigated for two length configurations: $645 \mathrm{~mm}$ (long) and $353 \mathrm{~mm}$ (short). Results of the investigation showed that although all the investigated geometries were converging nozzles formed by rotation of smooth meridional curve, there are significant differences in the flow field.

The individual nozzles exhibited differences in boundary layer growth, in kinetic energy losses, and in velocity profile at the end of the nozzle. Long configurations proved to have more uniform flow field but also higher losses and thicker boundary layer. Short configurations proved to have higher differences between velocity at the axis and near the wall but had thinner boundary layer and lower losses of kinetic energy. The shorter configuration of the two-sine nozzle might be more prone to flow separation due to very sharp change of curvature near the initial contraction, effectively forming a corner.

The long configuration of the Vagt nozzle was determined to be optimal thanks to the zero curvature at the end and uniform velocity profile. Relatively higher losses were not deemed as major drawback for intended application.

\section{Acknowledgement}

This study was made possible thanks to the support by the Czech Science Foundation under the grant number $20-11537 \mathrm{~S}$. Authors would also like to express their gratitude to Dr. Pavel Jonáš who on many occasions was kindly willing to share his knowledge on the subject.

\section{References}

[1] M. Deich: Technical gas dynamic, Moscow, Leningrad, Gousudarstvennoye Energeticheskoye Izdatel'stvo (1961).

[2] D. Hanus: Experimentální vzduchová trat' pro výzkum v zakřivených kanálech.Vnitřní aerodynamika lopatkových strojů.

[3] J.-D. Vagt: Bemerkungen zur Aslegung eines Unterschall-Freistrahlwindkanals, Zeitschrift für Flugwissenschaftten, vol. 5, no. 21, pp. 159-162 (1973).

[4] ANSYS, ANSYS CFX-Solver Theory Guide, (2021).

[5] F. Menter, J. Ferreira, T. Eash and B. Konno: The SST turbulence model with improved wall treatment for heat transfer predictions in gas turbines, Proceedings of the International Gas Turbine Congress, pp. 2-7 (2003).

[6] A. H. Shapiro: The Dynamics and Thermodynamics of Copressible Fluif Flow, New York: The Ronald press company (1953). 